\title{
Design and simulation of one-port SAW resonator for wireless and high temperature application
}

\begin{abstract}
This paper present the full design consideration, operational principle, structure and frequency response simulation of one-port Surface Acoustic Wave (SAW) Resonator using Micro Wave Office (MWO) and MATLAB used for passive wireless sensing of various measurands. Since SAW Resonators are widely used in radio frequency sensing application, the development and evolution of present day SAW devices has been possible only due to simultaneous efforts to simulate these devices and also it is desirable to evaluate the device prior to fabrication especially in cases where the characteristic of device material and dimensions have a major influence on the performance of the overall system. The equivalent circuit model approach has been used to simulate the frequency response of one-port SAW resonator base on gallium orthophosphate (GaPO4) with 5deg cut-angle at $433.92 \mathrm{MHz}$ under ISM band frequency for high temperature wireless application and it appears that this modeling is suitable for near resonance frequency, also measuring dielectric permittivity of GaPO4 at this frequency for use of modeling.
\end{abstract}

